Western University

Scholarship@Western

Bone and Joint Institute

$1-1-2015$

\title{
A comparison of registration errors with imageless computer navigation during MIS total knee arthroplasty versus standard incision total knee arthroplasty: A cadaveric study
}

\author{
Edward T. Davis \\ The Royal Orthopaedic Hospital NHS Foundation Trust \\ Joseph Pagkalos \\ The Royal Orthopaedic Hospital NHS Foundation Trust \\ Price A. Price \\ Gold Coast Hospital \\ Kelly Macgroarty \\ Brisbane Private Hospital \\ James P. Waddell \\ Saint Michael's Hospital University of Toronto
}

See next page for additional authors

Follow this and additional works at: https://ir.lib.uwo.ca/boneandjointpub

Part of the Medicine and Health Sciences Commons

Citation of this paper:

Davis, Edward T.; Pagkalos, Joseph; Price, Price A.; Macgroarty, Kelly; Waddell, James P.; and Schemitsch, Emil H., "A comparison of registration errors with imageless computer navigation during MIS total knee arthroplasty versus standard incision total knee arthroplasty: A cadaveric study" (2015). Bone and Joint Institute. 1050.

https://ir.lib.uwo.ca/boneandjointpub/1050 
Authors

Edward T. Davis, Joseph Pagkalos, Price A. Price, Kelly Macgroarty, James P. Waddell, and Emil H. Schemitsch 


\section{A comparison of registration errors with imageless computer navigation during MIS total knee arthroplasty versus standard incision total knee arthroplasty: a cadaveric study}

Edward T. Davis, Joseph Pagkalos, Price A. M. Gallie, Kelly Macgroarty, James P. Waddell \& Emil H. Schemitsch

To cite this article: Edward T. Davis, Joseph Pagkalos, Price A. M. Gallie, Kelly Macgroarty, James P. Waddell \& Emil H. Schemitsch (2015) A comparison of registration errors with imageless computer navigation during MIS total knee arthroplasty versus standard incision total knee arthroplasty: a cadaveric study, Computer Aided Surgery, 20:1, 7-13, DOI: 10.3109/10929088.2015.1076037

To link to this article: https://doi.org/10.3109/10929088.2015.1076037

а (C) 2015 The Author(s). Published by Taylor \& Francis.

Submit your article to this journal $\asymp$

\section{ए8}

Published online: 20 Aug 2015. submit your article to this journal

View related articles

View Crossmark data

Citing articles: 1 View citing articles 


\title{
A comparison of registration errors with imageless computer navigation during MIS total knee arthroplasty versus standard incision total knee arthroplasty: a cadaveric study*
}

\author{
Edward T. Davis ${ }^{a}$, Joseph Pagkalos ${ }^{a}$, Price A. M. Gallie ${ }^{b}$, Kelly Macgroarty ${ }^{c}$, James P. Waddell ${ }^{d}$, \\ and Emil H. Schemitsch ${ }^{\mathrm{d}}$ \\ ${ }^{a}$ The Royal Orthopaedic Hospital NHS Foundation Trust, Northfield, Birmingham, UK, b Gold Coast Hospital, Queensland, Australia, \\ 'Brisbane Private Hospital, Brisbane, Australia, and ${ }^{\mathrm{d} S}$ St Michael's Hospital, University of Toronto, Toronto, Ontario, Canada
}

ABSTRACT

Optimal component alignment in total knee arthroplasty has been associated with better functional outcome as well as improved implant longevity. The ability to align components optimally during minimally invasive (MIS) total knee replacement (TKR) has been a cause of concern. Computer navigation is a useful aid in achieving the desired alignment although it is limited by the error during the manual registration of landmarks. Our study aims to compare the registration process error between a standard and a MIS surgical approach. We hypothesized that performing the registration error via an MIS approach would increase the registration process error. Five fresh frozen lower limbs were routinely prepared and draped. The registration process was performed through an MIS approach. This was then extended to the standard approach and the registration was performed again. Two surgeons performed the registration process five times with each approach. Performing the registration process through the MIS approach was not associated with higher error compared to the standard approach in the alignment parameters of interest. This rejects our hypothesis. Image-free navigated MIS TKR does not appear to carry higher risk of component malalignment due to the registration process error. Navigation can be used during MIS TKR to improve alignment without reduced accuracy due to the approach.
ARTICLE HISTORY

Received 13 September 2013

Accepted 2 July 2015

\section{KEYWORDS}

Alignment; arthroplasty; imageless navigation, registration error; total knee replacement

\section{Introduction}

Minimally invasive (MIS) total knee replacement (TKR) was developed as a sequel of MIS techniques for unicompartmental knee arthroplasty.[1] Whilst initially defined as a knee replacement via an incision of $14 \mathrm{~cm}$ or less, the definition expanded to include the extent of injury to the structures surrounding the involved joint.[2] To classify a knee replacement as MIS, the amount of soft tissue damage (muscle, ligament and capsule), patellar eversion or retraction and dislocation of the joint should all be taken into account.[3]

The ability to align the components optimally in MIS TKR has been a source of concern. Radiographic complications in the form of component malalignment or malpositioning in MIS TKR have been reported between $1 \%$ and $20 \%$ in a review of RCT and retrospective cohorts.[4-6] The combination of computer navigation with MIS aims to provide optimal component positioning whilst achieving the benefits of the less invasive technique. Two recent trials reported improved coronal plane alignment of the components within $3^{\circ}$ when comparing computer navigated MIS TKR with standard TKR [7] or nonnavigated MIS TKR.[8]

During image-free computer navigation, the operating surgeon has to register several anatomical landmarks. The error during this registration process has been a cause of concern; significant error could lead to suboptimal component alignment.[9-13] Component malalignment has been identified as a cause for adverse functional outcomes $[14,15]$ and compromised longevity $[16,17]$ in TKR.

The aim of this study was to define the error during the registration process for computer navigated MIS TKR and compare this with the error during registration for standard incision navigated TKR. The hypothesis was that performing the registration process via an MIS approach would increase the error compared to the standard incision.

*The research took place at St. Michael's Hospital, University of Toronto, Toronto, Ontario, Canada.

Correspondence: Edward T. Davis, MBChB, MSc, FRCS (T\&O), Consultant Surgeon, The Royal Orthopedic Hospital NHS Foundation Trust, Northfield B31 2AP Birmingham, UK. eddavis@doctors.org.uk

(C) 2015 The Author(s). Published by Taylor \& Francis. This is an Open Access article distributed under the terms of the Creative Commons Attribution License (http://creativecommons.org/Licenses/by/4.0/), which permits unrestricted use, distribution, and reproduction in any medium, provided the original work is properly cited. 


\section{Methods}

Five fresh frozen cadaveric limbs including the hemipelvis were used for this study. The cadavers were sectioned through the midline of the pelvis and were secured to the table using G-clamps. The limbs were draped as per standard TKR without the use of a tourniquet. The ankle and foot were not draped. A MIS (mini mid-vastus) approach was performed with the patella subluxed and with dislocation of the joint. The navigation system used was the Stryker Knee Navigation System v. 2.0(Stryker Navigation, Kalamazoo, MI). Two surgeons registered the anatomical landmarks of each specimen five times via this approach. The approach was then extended to a standard incision for a TKR with a medial parapatellar arthrotomy and the two surgeons repeated the registration process five times. In order to define the gold standard of each anatomical point, the soft tissues were stripped and the bony landmarks were registered by the senior author (E.S.). The error during the kinematic registration of the hip centre significantly affected the mechanical axis error. This was thought to be due to the movement allowed at the fixation of the hemi-pelvis to the G-clamp. To eliminate this, the mechanical axis error in the femur was calculated using simple trigonometry using a fixed length for the distance from the hip centre to the epicondyles of $38 \mathrm{~cm}$.[18]

\section{Statistical analysis}

The data were assessed for normality using the ShapiroWilk test. Significance was tested using the paired $t$-test and the Wilcoxon signed-rank test for parametric and non-parametric data accordingly. Statistical analysis was performed using SPSS statistics 17.0.1 (SPSS Inc., Chicago, IL). A post hoc power analysis was performed. The sample size of our study would provide $80 \%$ power at an alpha level of 0.05 to identify a difference of $2.2^{\circ}$ of rotation of the transepicondylar axis, $0.3^{\circ}$ of error of the tibial mechanical axis in the coronal plane and $0.2^{\circ}$ of error in the femoral mechanical axis in the coronal plane.

\section{Results}

The minimum and maximum error in the registration of each landmark and the resulting error in calculation of the relevant axes are presented in Table I.

A statistically significant difference was identified between the approaches for the landmarks: centre of femur (sagittal), lateral epicondyle (sagittal), ankle centre (coronal) as well as the mechanical axis or the femur (sagittal) $(p<0.05)$. The error range during the registration of the lateral epicondyle (sagittal) and the centre of the femur (coronal and sagittal) was greater during the standard approach compared to the MIS approach. The error in the registration of the centre of the femur is presented in Figure 1. The resulting error in the calculation of the femoral mechanical axis is demonstrated in Figure 2. The error in the registration of the tibia landmarks is presented in Figure 3 and the resulting error in calculation of the mechanical axis in the coronal and sagittal plane is demonstrated in Figure 4. The error in the registration of the femoral epicondyles and the calculation of the transepicondylar axis is demonstrated in Figures 5 and 6.

\section{Discussion}

Our study has shown that the difference in the error during the registration process for image-free computer navigation through MIS and standard approaches did not reach statistical significance for the alignment parameters of interest. The mechanical axis of the femur and tibia in the coronal plane as well as the transepicondylar axis did not demonstrate statistically

Table I. The error during registration of each anatomical landmark using each approach.

\begin{tabular}{|c|c|c|c|c|c|c|c|c|}
\hline \multirow[b]{2}{*}{ Landmark } & \multirow[b]{2}{*}{ Plane } & \multicolumn{3}{|c|}{ MIS } & \multicolumn{3}{|c|}{ Standard } & \multirow[b]{2}{*}{$p$ Value } \\
\hline & & Mean & SD & Range & Mean & SD & Range & \\
\hline Distal femur centre & Coronal & -0.8 & 2.2 & -4.9 to 3.1 & 0.0 & 3.0 & -6.5 to 5.0 & $0.415^{\mathrm{a}}$ \\
\hline Distal femur centre & Sagittal & -4.6 & 2.3 & -9.2 to 0.2 & -6.9 & 3.3 & -13.2 to -0.2 & $0.000^{\mathrm{b}}$ \\
\hline Femur mechanical axis & Coronal & 0.0 & 0.3 & -0.7 to 0.5 & 0.0 & 0.5 & -1.0 to 0.8 & $0.415^{\mathrm{a}}$ \\
\hline Femur mechanical axis & Sagittal & -0.7 & 0.3 & -1.4 to 0.0 & -1.0 & 0.5 & -2.0 to -0.2 & $0.000^{\mathrm{b}}$ \\
\hline Lateral epicondyle & Sagittal & -1.0 & 2.6 & -6.9 to 4.9 & -2.5 & 2.7 & -8.1 to 6.3 & $0.006^{b}$ \\
\hline Medial epicondyle & Sagittal & 2.1 & 6.5 & -8.9 to 12.6 & 2.1 & 6.5 & -8.9 to 12.6 & $0.612^{a}$ \\
\hline Epicondylar axis & Internal rotation & -2.1 & 4.1 & -10.3 to 6.5 & -3.1 & 3.6 & -10.3 to 4.8 & $0.161^{b}$ \\
\hline Proximal tibia centre & Coronal & 3.2 & 2.3 & -0.5 to 7.9 & 3.2 & 2.2 & -2.3 to 9.9 & $0.950^{\mathrm{a}}$ \\
\hline Proximal tibia centre & Sagittal & 3.3 & 3.2 & -3.1 to 8.4 & 3.4 & 2.3 & -0.6 to 9.6 & $0.841^{\mathrm{b}}$ \\
\hline Ankle centre & Coronal & 0.9 & 2.6 & -5.0 to 6.1 & -0.4 & 2.8 & -6.9 to 4.7 & $0.029^{b}$ \\
\hline Ankle centre & Sagittal & -4.0 & 4.2 & -14.1 to 3.6 & -3.7 & 3.7 & -12.9 to 3.7 & $0.754^{\mathrm{b}}$ \\
\hline Tibia mechanical axis & Coronal & 0.4 & 0.6 & -0.7 to 1.5 & 0.6 & 0.6 & -1.0 to 1.6 & $0.090^{\mathrm{b}}$ \\
\hline Tibia mechanical axis & Sagittal & -1.1 & 1.0 & -2.9 to 0.6 & -1.1 & 0.8 & -3.2 to 0.6 & $0.950^{\mathrm{a}}$ \\
\hline
\end{tabular}

${ }^{a}$ Wilcoxon signed-rank test.

${ }^{\mathrm{b}}$ Paired $t$-test. 
Figure 1. Boxplot of the error in the registration of anatomical landmarks in the femur. •: Outliers between $\times 1.5$ and $\times 3$ the interquartile range. ${ }^{*} p<0.05$. MIS, minimally invasive approach; STD, standard approach.
Figure 2. Boxplot of the error in calculation of the mechanical axis of the femur. $\bullet$ : Outliers between $\times 1.5$ and $\times 3$ the interquartile range. ${ }^{*} p<0.05$. MIS, minimally invasive approach; STD, standard approach.
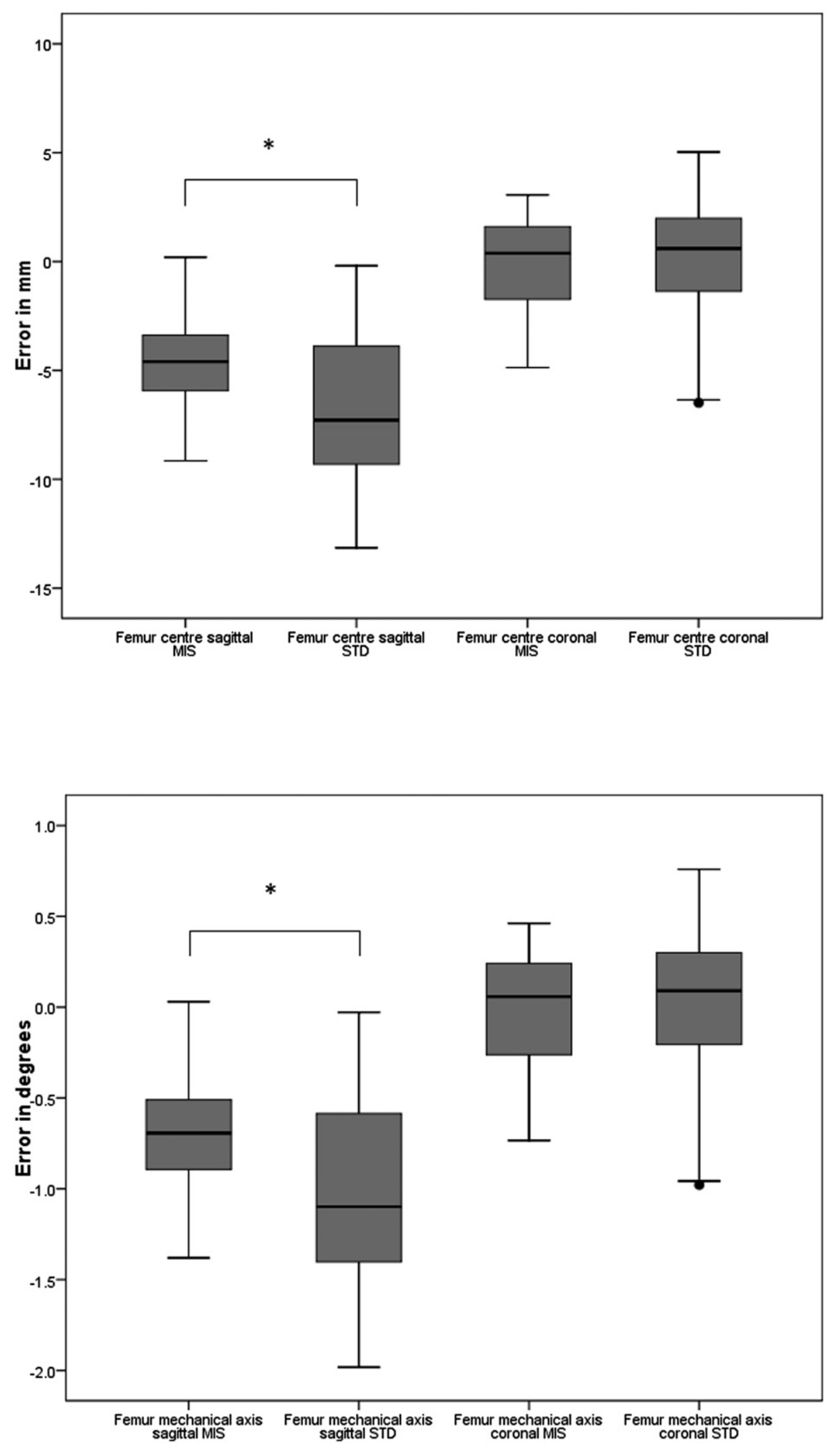

significant differences between the two approach groups. Our study was adequately powered to identify differences in the mechanical axes of the tibia and femur (coronal plane) of less than $0.5^{\circ}$. For the transepicondylar axis, the power of the study was adequate to identify a difference of $2.2^{\circ}$ (80\% power, alpha 0.05). A difference smaller than $2.2^{\circ}$ could remain undetected by the current study and would require larger sample size. 
Figure 3. Boxplot of the error in the registration of anatomical landmarks in the tibia and ankle. •: Outliers between $\times 1.5$ and $\times 3$ the interquartile range. ${ }^{*}: p<0.05$. MIS, minimally invasive approach; STD, standard approach.

Figure 4. Boxplot of the error in calculation of the mechanical axis of the tibia. $\bullet$ : Outliers between $\times 1.5$ and $\times 3$ the interquartile range. MIS, minimally invasive approach; STD, standard approach.
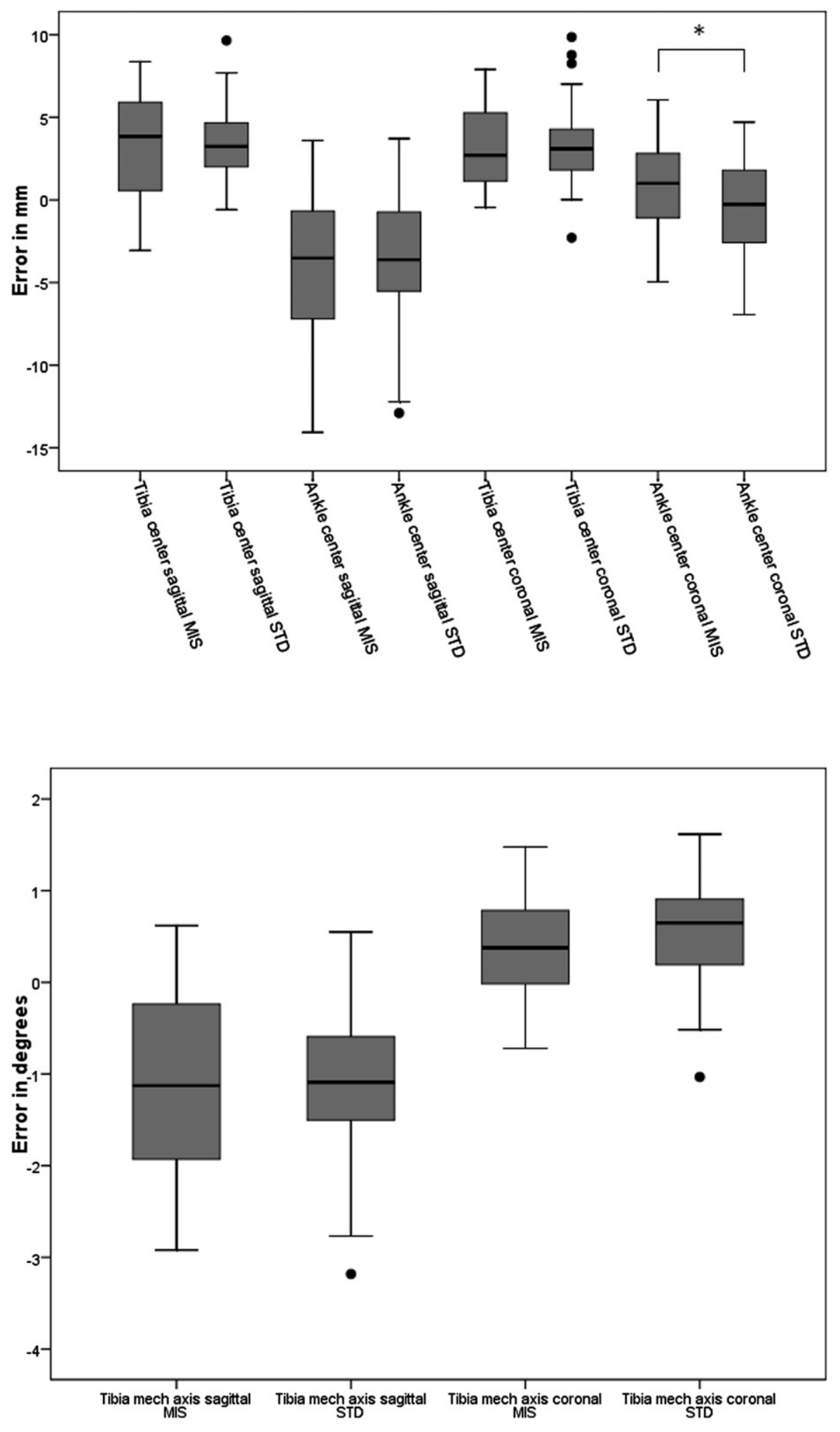

Consistently, through the MIS and standard approaches, the error range in defining the transepicondylar axis was greater when compared to that of the mechanical axis of the tibia and femur.
A significant error in the registration of the hip centre was documented despite the use of a pelvic pin. This was believed to be due to the fixation of the hemipelvis using $\mathrm{G}$ clamps that allowed for a degree of movement. 
Figure 5. Boxplot of the error in the registration of the epicondyles.

$\bullet$ : Outliers between $\times 1.5$ and $\times 3$ the interquartile range. ${ }^{*}: p<0.05$. MIS, minimally invasive approach; STD, standard approach.
Figure 6. Boxplot of the error in the calculation of the transepicondylar axis. MIS, minimally invasive approach; STD, standard approach.
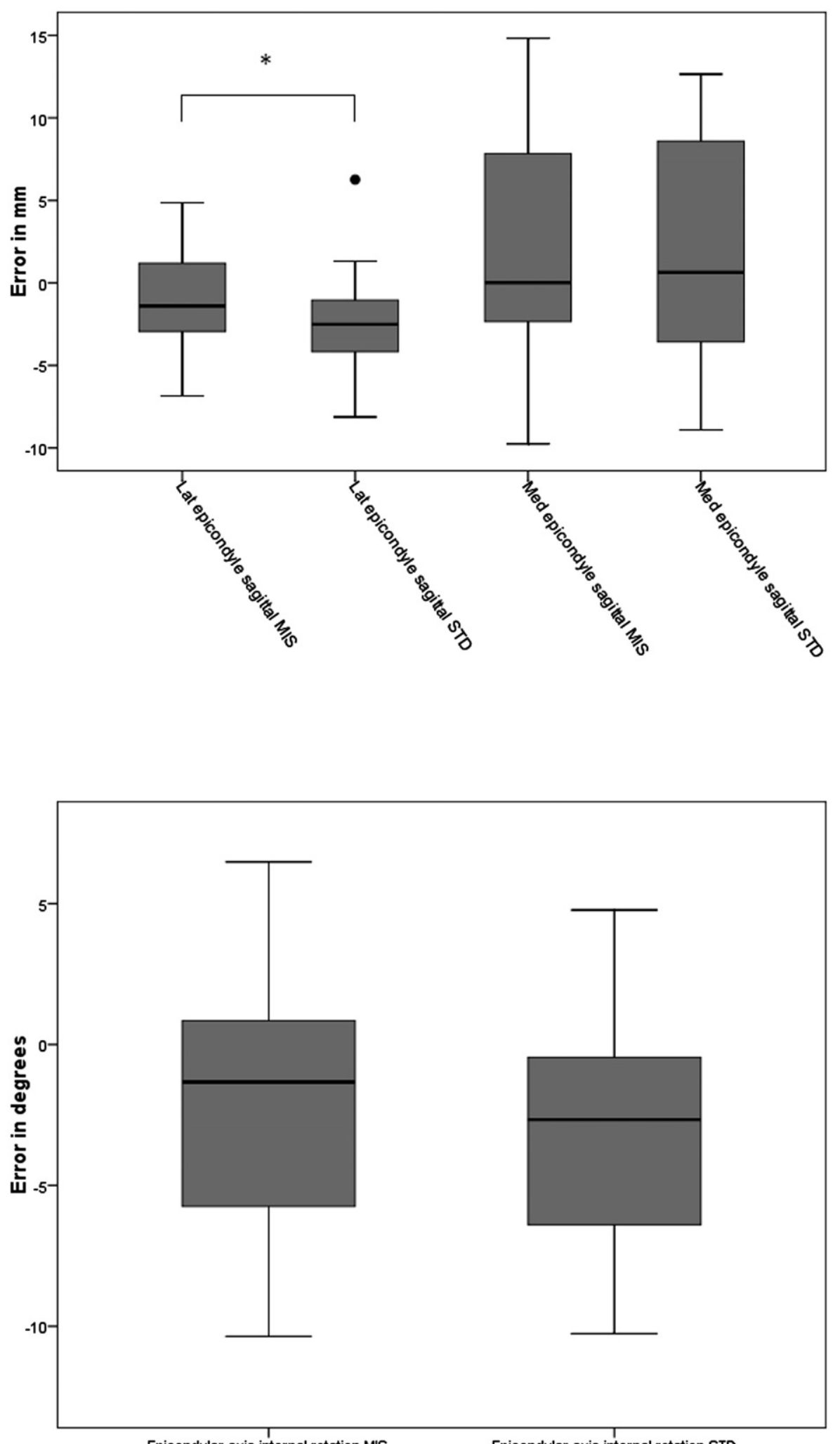

Epicondylar axis internal rotation MIS
To eliminate this, we calculated the femoral mechanical axis error from the error of registration of the centre of the distal femur and a fixed femur length using simple trigonometry.
For the MIS approach, the epicondylar axis mean error was $-2.1^{\circ}$ of internal rotation (SD 4.1) when the mean error in defining the mechanical axis of the femur was 0 degrees of varus (SD 0.3). During the standard 
approach, the epicondylar axis error was $-3^{\circ}$ of internal rotation (SD 3.6) and the femur mechanical axis error was $0^{\circ}$ of varus (SD 0.5).

The error in defining the transepicondylar axis via the standard approach has been previously reported. Siston et al. reported a mean error of $5.4^{\circ}$ (SD 7.1) during the calculation of the axis.[11] A previous cadaveric study comparing the error during the registration process for MIS and standard approaches showed similar magnitude of error when calculating the transepicondylar axis. The mean error was $4.5^{\circ}$ of internal rotation during MIS (SD 4) and $3.0^{\circ}$ (SD 4) during the standard approach $(p<0.01)$. The error in calculating the mechanical axis was not addressed during that study. A main difference between our study and that of Yau et al. is that the authors of the previous study used two dimensional CT scanning to define the gold standard transepicondylar axis.[13] During our study, the gold standard was the point registered by the senior author after stripping the soft tissues off the specimen. Defining the transepicondylar axis using plain film and two dimensional CT scanning was previously shown to carry significant risk of error as the operator has to pick the most prominent point of the epicondyle.[19] This may be affected by the rotation of the limb (plain film) and the axis of the slice (CT). Recent CT protocols have been developed that use $3 \mathrm{D}$ reconstruction to identify the alignment parameters with limited radiation exposure.[20,21] The use of imaging to define the gold standard also provides an error range between the manually selected points and the radiologically selected point. The different methodologies utilized in the aforementioned studies highlight the lack of agreement as to what is the gold standard point. That is a fundamental problem of accuracy studies. We therefore opted to focus on the precision of the manually selected points. In our study, the errors reported were those between the registration point obtained before and after stripping of the soft tissues, giving an estimation of the error range encountered during the manual registration process.

A number of randomized controlled trials have compared MIS TKR with the standard approach. A recent meta-analysis that pooled data from nine RCTs revealed an increased incidence of local surgical complications in the MIS group compared to standard TKR.[22] The authors commented on the fact that only four of nine studies stated the surgeon's experience prior to commencing the study. The learning curve effect has previously been documented with the early cases having longer operative time and suboptimal radiographic outcome.[23]
Malalignment of the mechanical axis in the coronal plane has been associated with poor long-term implant survival [17] as well as suboptimal functional outcome.[14] Rotational malalignment is associated with condylar lift off and patellar maltracking.[24,25] Concerns have been raised regarding the ability to align the implants appropriately during MIS TKR. Studies have reported increased risk of anatomical outliers with quadriceps sparing MIS,[26] whilst concerns about early revision of MIS knee replacements were documented by analysis of a consecutive revision TKR series by five surgeons.[6] The combination of computer navigation and MIS surgery has shown promising results. Dutton et al. demonstrated shorter inpatient stay, better function at one month and improved implant alignment in the navigated MIS group compared to standard TKR.[27] A further study by Hasegawa et al. comparing MIS with navigated MIS TKR showed improved alignment in the coronal plane $\left( \pm 3^{\circ}\right.$ from neutral) with no change in function (Knee Society Score), range of movement and other alignment factors.[8]

This study aimed to address the error in the registration process during MIS computer navigated TKR and compare it with that of the standard navigated technique. Defining the gold standard anatomical points using the navigation system after the soft tissue stripping allowed for a more accurate representation of the error range during the process. Performing the registration process through the MIS approach was not associated with greater error than during the standard approach. Therefore, we conclude that imagefree navigated MIS TKR does not carry a higher risk of component malalignment due to registration process error.

\section{Declaration of interest}

E.H.S. reports personal fees and other from Stryker, personal fees and other from Smith\&Nephew, personal fees from Amgen, personal fees from Wright medical, other from Zimmer, other from Synthes, other from Sanofi, grants from $\mathrm{CiHR}$, grants from PSI, grants from OTA, grants from $A O$, personal fees from Elsevier, outside the submitted work; P.A.G. reports personal fees and non-financial support from Smith\&Nephew, outside the submitted work; E.T.D. reports grants and personal fees from Brainlab, outside the submitted work. The other authors report no conflicts of interest.

\section{References}

1. Repicci JA, Eberle RW. Minimally invasive surgical technique for unicondylar knee arthroplasty. J South Orthop Assoc 1999;8:20-27; discussion 27.

2. Tria AJ Jr. Advancements in minimally invasive total knee arthroplasty. Orthopedics 2003;26:s859-s863. 
3. Bonutti PM, Mont MA, McMahon $M$, et al. Minimally invasive total knee arthroplasty. J Bone Joint Surg Am 2004;86-A:26-32.

4. Chin PL, Foo LS, Yang KY, et al. Randomized controlled trial comparing the radiologic outcomes of conventional and minimally invasive techniques for total knee arthroplasty. J Arthroplasty 2007;22:800-806.

5. Pagnano MW, Meneghini RM. Minimally invasive total knee arthroplasty with an optimized subvastus approach. J Arthroplasty 2006;21:22-26.

6. Barrack RL, Barnes $\mathrm{CL}$, Burnett RS, et al. Minimal incision surgery as a risk factor for early failure of total knee arthroplasty. J Arthroplasty 2009;24:489-498.

7. Dutton $A Q$, Yeo SJ, Yang KY, et al. Computer-assisted minimally invasive total knee arthroplasty compared with standard total knee arthroplasty. A prospective, randomized study. J Bone Joint Surg 2008;90:2-9.

8. Hasegawa M, Yoshida K, Wakabayashi $H$, Sudo A. Minimally invasive total knee arthroplasty: comparison of jig-based technique versus computer navigation for clinical and alignment outcome. Knee Surg Sports Traumatol Arthrosc 2011;19:904-910.

9. Jenny JY, Boeri C. Low reproducibility of the intraoperative measurement of the transepicondylar axis during total knee replacement. Acta Orthop Scand 2004;75:74-77.

10. Robinson $M$, Eckhoff $D G$, Reinig $K D$, et al. Variability of landmark identification in total knee arthroplasty. Clin Orthop Relat Res 2006;442:57-62.

11. Siston RA, Patel JJ, Goodman SB, et al. The variability of femoral rotational alignment in total knee arthroplasty. J Bone Joint Surg Am 2005;87:2276-2280.

12. Yau W, Leung A, Liu K, et al. Interobserver and intraobserver errors in obtaining visually selected anatomical landmarks during registration process in non-image-based navigation-assisted total knee arthroplasty. J Arthroplasty 2007;22:1150-1161.

13. Yau WP, Leung A, Liu KG, et al. Errors in the identification of the transepicondylar and anteroposterior axes of the distal femur in total knee replacement using minimallyinvasive and conventional approaches: a cadaver study. J Bone Joint Surg Br 2008;90:520-526.

14. Longstaff LM, Sloan K, Stamp N, et al. Good alignment after total knee arthroplasty leads to faster rehabilitation and better function. J Arthroplasty 2009;24: 570-578.

15. Nicoll D, Rowley DI. Internal rotational error of the tibial component is a major cause of pain after total knee replacement. J Bone Joint Surg Br 2010;92: 1238-1244.

16. Berend $M E$, Ritter MA, Meding JB, et al. Tibial component failure mechanisms in total knee arthroplasty. Clin Orthop Relat Res 2004;428:26-34.

17. Jeffery RS, Morris RW, Denham RA. Coronal alignment after total knee replacement. J Bone Joint Surg $\mathrm{Br}$ 1991;73:709-714.

18. Luo W, Stanhope SJ, Sheehan FT. Using two palpable measurements improves the subject-specific femoral modeling. J Biomech 2009;42:2000-2005.

19. Hirschmann MT, Konala $P, A m s l e r ~ F$, et al. The position and orientation of total knee replacement components: a comparison of conventional radiographs, transverse 2D$\mathrm{CT}$ slices and 3D-CT reconstruction. J Bone Joint Surg Br 2011;93:629-633.

20. Chauhan SK, Clark GW, Lloyd S, et al. Computer-assisted total knee replacement. A controlled cadaver study using a multi-parameter quantitative $\mathrm{CT}$ assessment of alignment (the Perth CT Protocol). J Bone Joint Surg $\mathrm{Br}$ 2004;86:818-823.

21. Henckel J, Richards R, Lozhkin K, et al. Very low-dose computed tomography for planning and outcome measurement in knee replacement. The imperial knee protocol. J Bone Joint Surg Br 2006;88:1513-1518.

22. Gandhi $R$, Smith $H$, Lefaivre KA, et al. Complications after minimally invasive total knee arthroplasty as compared with traditional incision techniques: a meta-analysis. J Arthroplasty 2011;26:29-35.

23. King J, Stamper DL, Schaad DC, Leopold SS. Minimally invasive total knee arthroplasty compared with traditional total knee arthroplasty. Assessment of the learning curve and the postoperative recuperative period. J Bone Joint Surg Am 2007;89:1497-1503.

24. Berger RA, Crossett LS, Jacobs JJ, Rubash HE. Malrotation causing patellofemoral complications after total knee arthroplasty. Clin Orthop Relat Res 1998;356:144-153.

25. Insall JN, Scuderi GR, Komistek RD, et al. Correlation between condylar lift-off and femoral component alignment. Clin Orthop Relat Res 2002;403:143-152.

26. Chen AF, Alan RK, Redziniak DE, Tria AJ Jr. Quadriceps sparing total knee replacement. The initial experience with results at two to four years. J Bone Joint Surg $\mathrm{Br}$ 2006;88:1448-1453.

27. Dutton AQ, Yeo SJ, Yang KY, et al. Computer-assisted minimally invasive total knee arthroplasty compared with standard total knee arthroplasty. A prospective, randomized study. J Bone Joint Surg Am 2008;90:2-9. 